\title{
84. ON A COROLLARY TO THE MAGNETIC DIPOLE THEORY OF THE ORIGIN OF SPIRAL STRUCTURE
}

\author{
PARIS PISMIS \\ Universidad Nacional Autónoma de México, Mexico City, Mexico
}

\begin{abstract}
Some observable features of our Galaxy are explained in terms of a theory of the origin of spiral structure, whose basic feature is a gradually contracting gaseous subsystem with a magnetic dipole of which the axis is very close to the galactic plane.
\end{abstract}

\section{Introduction}

The aim of this communication is to present a few consequences (a corollary) of a theory, which was advanced some years ago by this author, to account for the origin and maintenance of the spiral structure in a galaxy. A series of ideas and arguments leading to the formulation of the theory are discussed in a number of papers published since 1960 (Pișmiş, 1960; Huang and Pişmiş, 1960; Pișmiș, 1961) while the outline of the theory itself with an application to the galaxy M31 has appeared in 1963 (Pișmiş, 1963). Here a brief sketch of this theory will be given prior to the presentation of a corollary to it.

\section{The Theory}

At present it is generally accepted that the stellar subsystems are formed at successive epochs in the course of the evolution of a galaxy. In a galaxy where the subsystems, from halo down to disk, are already formed, we assume that there still remains an organized gaseous subsystem, a flat spheroid, concentric with the galaxy at large and that this gaseous subsystem possesses, and is held together by, a magnetic field which, as a rough first approximation, is taken to be a dipole. We postulate that the magnetic dipole is centrally located and its axis is perpendicular to the axis of rotation of the galaxy. Such an orientation for the dipole field is intuitively quite an unorthodox assumption but herein lies the novelty of our approach.

It is expected that due to infinite conductivity of the interstellar gas the spheroid will rotate as a rigid body. No distortion of the dipole field inside the spheroid should thus occur. Next, it is plausible that the gaseous spheroid will suffer a gradual contraction and during this process matter and field will contract together. The only regions from where gas may leak out are those of the magnetic poles. The locus of the gas thus left behind will be a spiral due to its slower rotation speed with respect to the contracting spheroid. (In a forthcoming paper a quantitative treatment of the loss of mass and angular momentum is given.)

An application of this mechanism to M31 using the observed rotation curve of this galaxy has yielded a pattern of two intertwined spirals, with 1.5 turns each, in $3 \times 10^{9}$ years (for details see Pișmiș, 1963). 
The gas may also leave the polar regions with a radial outward velocity, in other words it may be ejected. It is easy to show that, be it with leakage or with ejection, a spiral pattern will ensue by the mechanism sketched above. It may thus be stated that as a consequence of this theory:

(a) A spiral gravitationed potential field is created and it is superposed on a smooth axis-symmetrical potential field of the previously existing stellar component of a galaxy.

(b) The bi-symmetry of the spiral pattern is accounted for.

(c) The magnetic field lines are clearly along the spiral pattern.

(d) Two consecutive arms in the grand spiral design are of opposite polarity.

It should be emphasized that the main function of the magnetic dipole field is to funnel the gas through two diametrically opposite points of the bulge and that, unlike previous magnetic theories, no account is taken of the magnetic force in the maintenance of the spiral forms. Although much remains to be done on the physics of the theory, interesting consequences emerge as a corollary to our proposition (Pișmiș, 1968).

\section{Corollary}

If the magnetic dipole is not exactly perpendicular to the rotation axis but deviates slightly from it, the magnetic field will change sign while crossing the plane of symmetry - the galactic plane - and two consecutive arms will be above and below the plane respectively.

To fix ideas assume, now, that the positive pole is slightly above the plane of symmetry of the Galaxy and the negative pole below the plane. The two sets of spirals engendered by the rotating dipole will no more be co-planar. The spiral produced by the positive pole will always remain above the plane and the diametrically opposite arm will always remain below the plane; therefore along a radius vector in the plane the arms will be seen alternately above and below the plane and their distance from the plane will increase as the edges of the galaxy are approached. This result seems to be supported by recent optical data and by the $21 \mathrm{~cm}$ surveys (Henderson, 1967). Moreover the sign of the magnetic field will be positive above and negative below the plane, a result also in line with recent observations showing a general reversal of the magnetic field while crossing the galactic plane.

One other consequence of the deviation from orthogonality of the dipole may be mentioned here. This is the increase of the overall width of the galactic stratum from the center towards the edge of a galaxy. According to recent knowledge (Henderson, 1967) the thickness of the neutral hydrogen layer in the Galaxy appears to vary monotonically from the center outwards; the thickness is about $250 \mathrm{pc}$ at the position of the sun and increases to about $500 \mathrm{pc}$ at the edge of the Galaxy. Taking account of the natural width of the arms and their irregularities it is not difficult to see that the combined width of the two sets of intertwined spirals may appear as a layer of neutral hydrogen which widens outwards.

An estimate of the angle of inclination of the magnetic dipole to the average galactic 
plane using the above estimates of the variation of thickness of the hydrogen layer has yielded a value of $45^{\prime}$ for this angle.

In concluding we may state that a simple corollary to our magnetic dipole theory for the origin of spiral structure appears to account for the following three phenomena suggested by observation, but apparently quite unrelated to one another, at first sight.

(1) The reversal of the magnetic field while crossing the galactic plane.

(2) The alternately up and down position of the consecutive spiral arms.

(3) The thickening of the neutral hydrogen layer towards the edges of the galaxy.

\section{References .}

Henderson, A. P.: 1967, Ph. D. Thesis, University of Maryland.

Huang, S. S. and Pişmiş, P.: 1960, Bol. Obs. Tonantzintla Tacubaya 2, 7.

Pişmiş, P.: 1960, Bol. Obs. Tonantzintla Tacubaya 2, 3.

Pişmiş, P.: 1961, Bol. Obs. Tonantzintla Tacubaya 3, 3.

Pişmiş, P.: 1963, Bol. Obs. Tonantzintla Tacubaya 3, 127.

Pişmiş, P.: 1968, Bol. Obs. Tonantzintla Tacubaya 4, 229. 